\title{
Calorimetric Calibration of an Ionization Chamber for Determination of X-ray Total Beam Energy*
}

\author{
John S. Pruitt and Steve R. Domen
}

(May 18, 1962)

\begin{abstract}
An X-ray calorimeter has been used to determine the total energy transported by bremsstrahlung beams with maximum photon energies between 18.2 and $170 \mathrm{Mev}$. The measurements from two experiments have been used to calibrate an aluminum ionization chamber for routine determinations of total beam energy. The calibrations are compared with calibrations of the same chamber made with a scintillation spectrometer, and with calorimetric calibrations made in other laboratories.
\end{abstract}

\section{Introduction}

Several techniques for the determination of the energy transported by a high energy X-ray beam have been described in the literature [1]. ${ }^{1}$ The most direct method is calorimetry, which can be used to determine the total beam energy without reference to the energy spectrum of the incident photons and without knowledge of the details of the calorimeter energy absorption processes. High energy X-ray calorimeter measurements have been reported by several authors $[2-5]$, and some of these measurements have been used to calibrate ionization chambers for routine determination of the total beam energy [3-5]. This paper is a description of an X-ray calorimeter and its use in two experiments to calibrate an aluminum ionization chamber. The determination of the fraction of energy escaping from the calorimeter is described in detail. Other calibrations of this same ionization chamber are reported and are shown to be in good agreement with the calibrations obtained in these experiments.

The first calorimeter experiment was performed with the NBS $50 \mathrm{Mev}$ betatron, using bremsstrahlung: beams with maximum photon energies between 18.2 and $42.1 \mathrm{Mev}$. The second experiment was performed with the NBS $180 \mathrm{Mev}$ synchrotron, covering the energy range between 20 and $170 \mathrm{Mev}$. The results of these two experiments have been combined to yield average calibrations with an estimated uncertainty of the order of \pm 2 percent between 20 and $170 \mathrm{Mev}$.

\section{Calorimeter}

Figure 1 is a schematic cross section of the X-ray calorimeter. The sensitive elements are the two cylinders, made of lead covered with thin gold-plated brass shells. The five concentric boxes and the thermoregulator provide a constant temperature environment for the cylinders, and precautions were taken to minimize heat exchange between the cylinders and the boxes. The highly polished gold surfaces of the cylinders and of box $\mathrm{A}$, designed to minimize thermal radiation, are shown in figure 2 .

*Supported in part by AEC.

1 Figures in brackets indicate the literature references at the end of this paper.
Exchange of energy by conduction and convection is practically eliminated by evacuating boxes $\mathrm{A}, \mathrm{B}$, and $\mathrm{C}$ and by mounting them, along with the cylinder, with thermally insulating plastics. The isolation of the sensitive elements is such that the thermal relaxation time of the cylinders is about $20 \mathrm{hr}$, almost an order of magnitude larger than the corresponding quantity reported for an earlier model [6].

Two 1,000 ohm bead thermistors with temperature coefficients of about -3.6 percent per ${ }^{\circ} \mathrm{C}$ and two 500 ohm carbon resistors are embedded in each cylinder. The four thermistors form the arms of a Wheatstone bridge, as shown in figure 3 . Their characteristics are not identical, but the bridge can be balanced by adjusting the length of the small external manganin wire resistor, $r$. Bridge power is supplied by a $1.34 \mathrm{v}$ mercury dry cell, and the output voltage between points $a$ and $b$ in the detection circuit is amplified by a breaker amplifier and displayed on a chart recorder. The circuit between amplifier and recorder adds a fixed d-c bias to the amplified signal to place the recorder pen at midscale when the amplifier input is shorted. This bias increases the average signal-tonoise ratio, for the noise was found to increase with increasing bridge unbalance.

The four carbon resistors are connected in two separate circuits, which can be used to heat the cylinders individually. The inner end of each resistor is in electrical contact with the cylinder, which forms an integral part of each circuit as shown by dashed lines in figure 3 .

The detection circuit output voltage is proportional to the temperature difference between the two cylinders, and can be used as a relative measure of heat generated in one of the cylinders, if corrections are made for heat lost to the surroundings. Figure 4 shows a chart record which was obtained with maximum amplifier gain when the source of heat was a $25 \mathrm{Mev}$ bremsstrahlung beam with a diameter of $4.2 \mathrm{~cm}$ and an intensity of $12 \mu \mathrm{w} / \mathrm{cm}^{2}$. The absolute temperature changes in figure 4 are only approximate and are shown for illustrative purposes only. The temperature changes are normally measured in chart divisions, and are converted directly into energy units with the calibrations described below, after the change caused by irradiation has been corrected for the average drift during irradiation. 


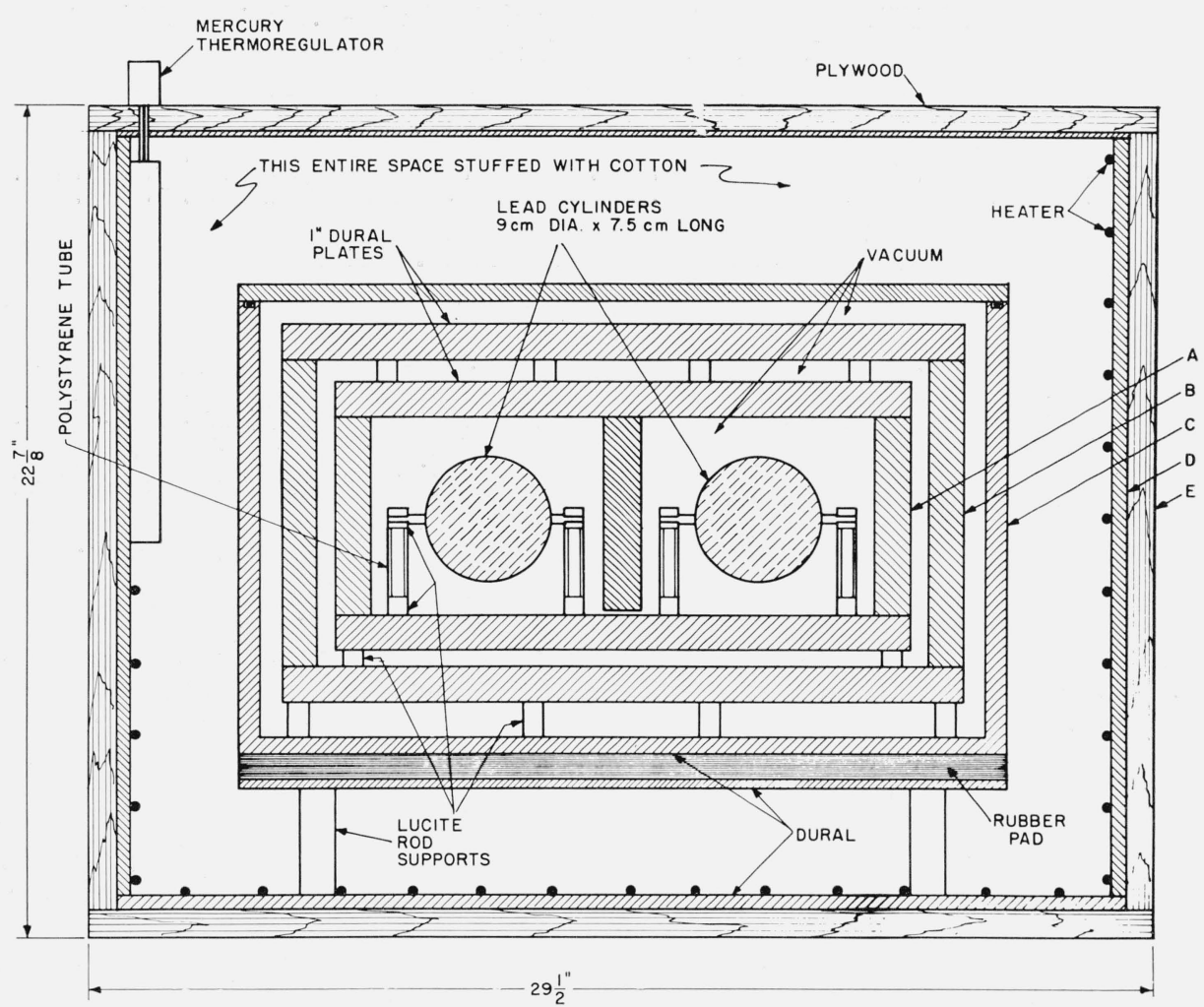

Figure 1. Cross section of X-ray calorimeter.

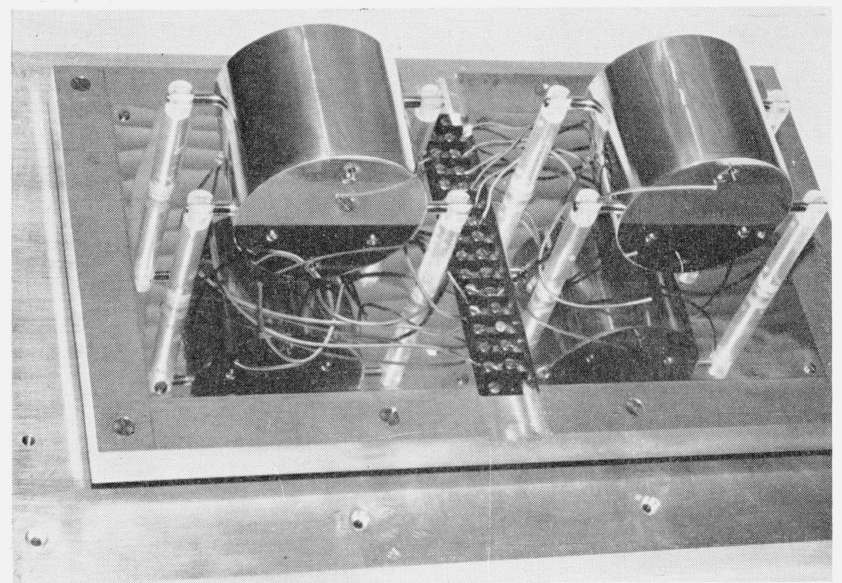

Figure 2. Calorimeter cylinders mounted on base plate of inner box.

The signal noise in figure 4 is typical for maximum gain operation and disappears when the amplifier input is shorted. The noise is less noticeable when a lower gain is used, which was feasible at higher energies where the available X-ray intensities were larger. This noise limits the precision of low energy calorimeter measurements, but these measurements are still reproducible with an rms deviation smaller than 4 percent even when the intensity is as low as $2 \mu \mathrm{w} / \mathrm{cm}^{2}$.
The recorder pen was returned to the bottom of the chart after each exposure in preparation for the next run, by heating the dummy cylinder with the compensation circuit of figure 3 . This was done in preference to adjusting the manganin wire, $r$, because the latter introduced spurious transients in the bridge output voltage which persisted for about $20 \mathrm{~min}$, and also increased the drift correction for succeeding runs. The manganin wire was changed only at the start of each day, to minimize the initial recorder pen drift rate.

The vertical scale of the chart recorder was calibrated after each X-ray exposure, to minimize errors introduced by changes in amplifier gain and by the gradual increase of the cvlinder temperatures during the course of a day. Calibration was performed by heating the previously irradiated cylinder electrically with a known power for a known time, using the calibration circuit of figure 3 . The power supplied by this circuit is the product of the voltages $V_{R}$ and $V_{C}$, which were both measured with a potentiometer, divided by the resistance of the precision wire wound resistor $R$. The net power dissipated in the cylinder was obtained by correcting for power lost in the cable (about $0.5 \%$ ), heat conducted away from the cylinder by the heater and thermistor leads $(0.1 \%)$, and heat radiated from the exposed ends of resistors $R_{3}$ and $R_{4}(0.1 \%)$. The power used for each calibration was preselected to approximate the X-ray power, to reduce the systematic errors. This preselection was done using the resistor $R^{\prime}$ in place of the cylinder. 


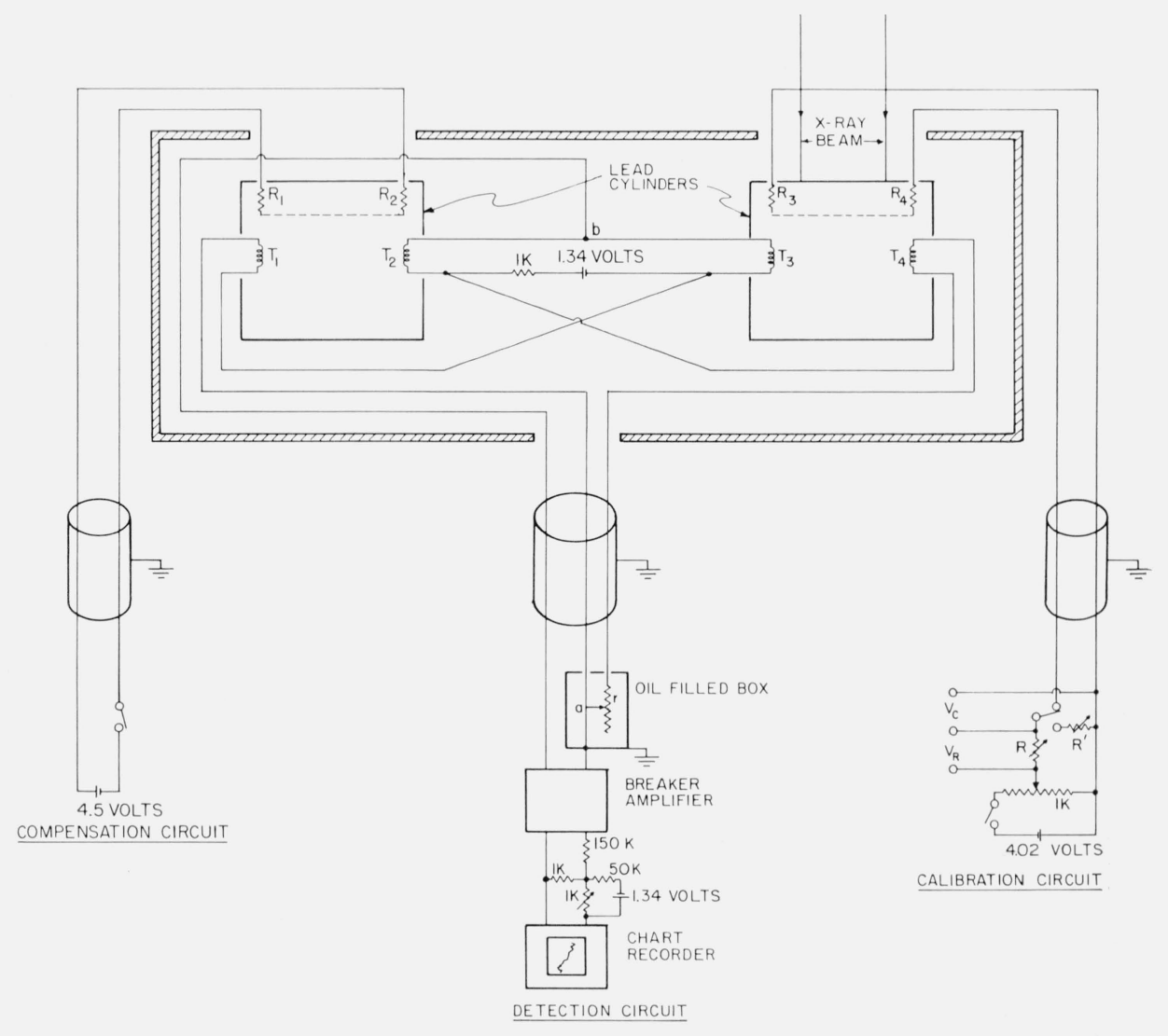

Figure 3. Schematic diagram of calorimeter circuits.

The $R_{i}$ are carbon resistors and the $T_{i}$ bead thermistors embedded in the calorimeter cylinders

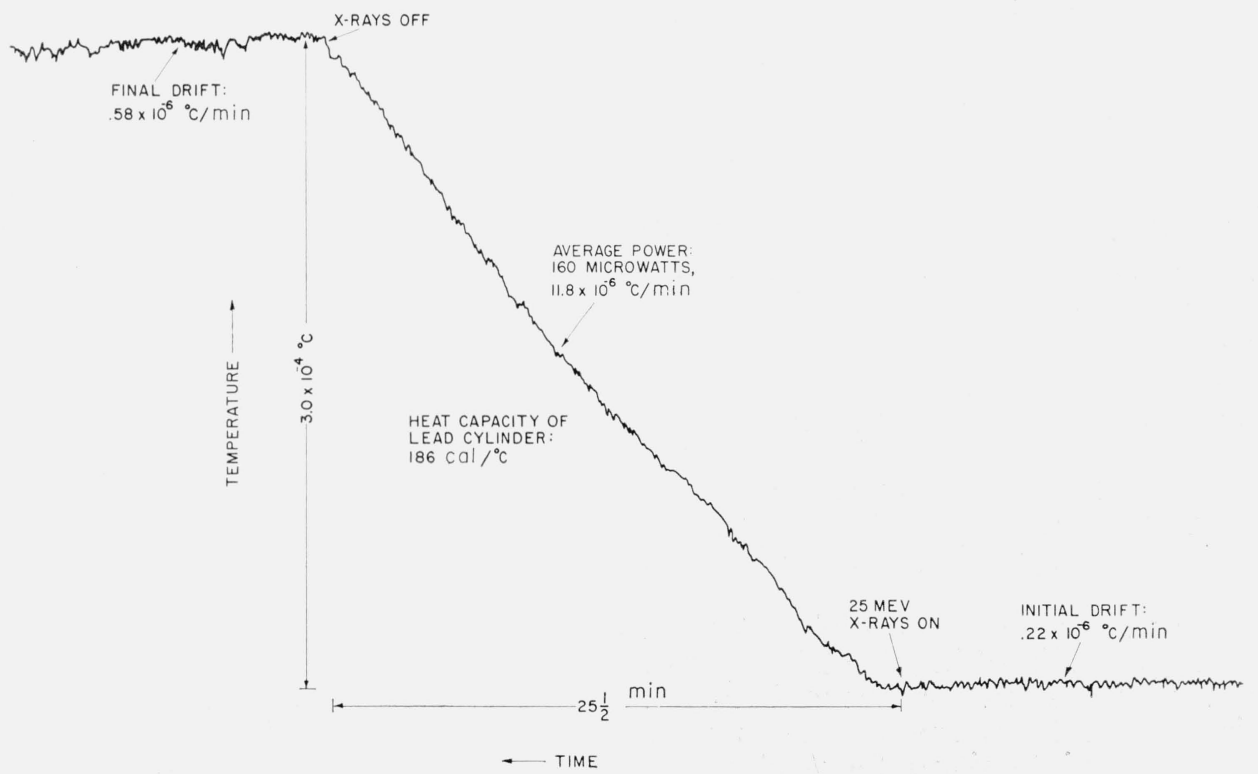

Figure 4. Typical chart record of an exposure to 25 Mev X-rays, using maximum amplifier gain. 


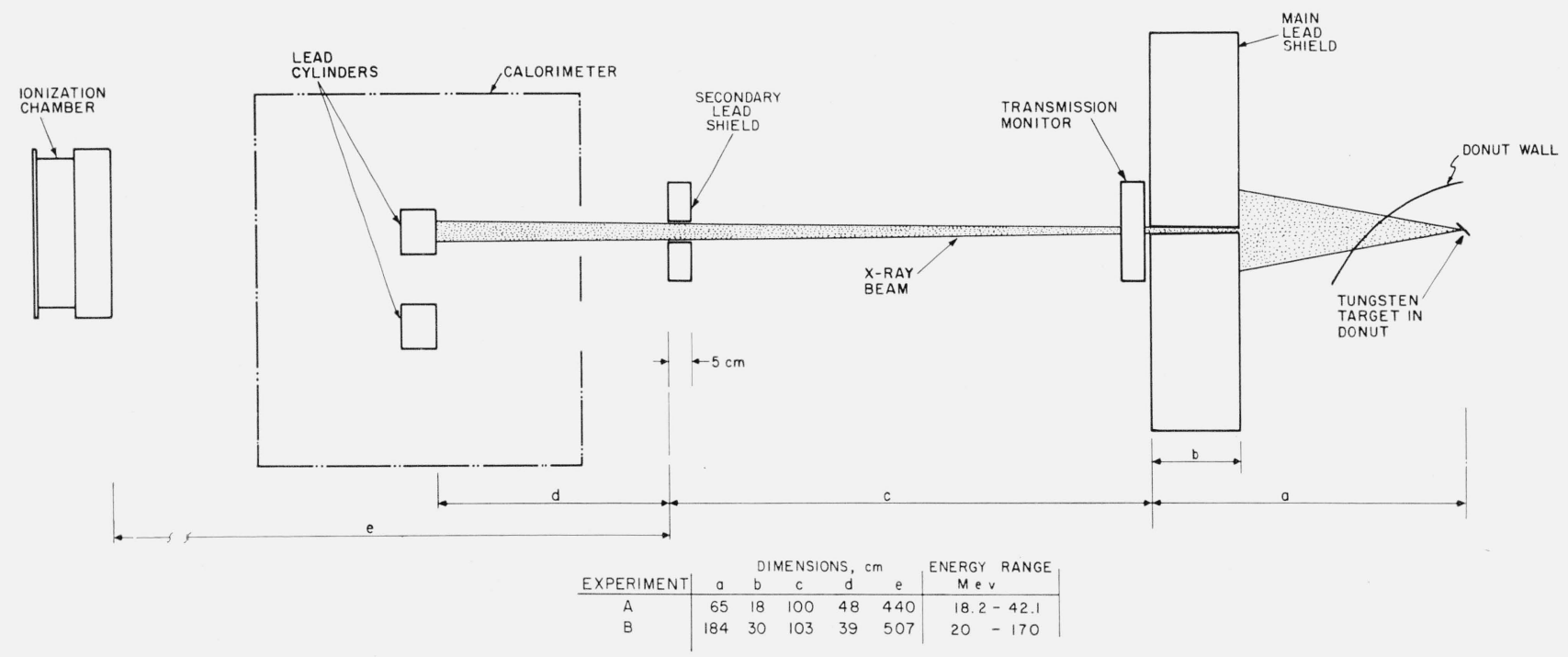

FiguRE 5. Experimental arrangement for the two calorimeter experiments.

\section{Calorimeter Measurements}

Figure 5 is a generalized schematic diagram of the experimental arrangements in the two calorimeter experiments. In experiment $A$, performed with the betatron, the bremsstrahlung beam was filtered by $1.6 \mathrm{~g} / \mathrm{cm}^{2}$ of porcelain in the wall of the electron acceleration chamber (the donut), and by $1.4 \mathrm{~g} / \mathrm{cm}^{2}$ of aluminum in transmission monitor A. In experiment $\mathrm{B}$, performed with the synchrotron, the filters were $4.1 \mathrm{~g} / \mathrm{cm}^{2}$ of Pyrex in the donut wall and 0.4 $\mathrm{g} / \mathrm{cm}^{2}$ of aluminum in monitor B. In each experiment, the main lead shield was chosen to produce a $4.2 \mathrm{~cm}$ diam beam at the calorimeter cylinder face, and the secondary lead shield was placed so that it could absorb extra-beam electrons and photons generated by interactions in the main shield and the monitor, without interfering with the direct beam.

Both transmission monitors were ionization chambers. Their stability was periodically checked, with the calorimeter removed, by comparison with the large ionization chamber shown in figure 5 and discussed in section 5. The total charge collected in each chamber during an X-ray exposure was measured in volts with a Townsend balance circuit, using a polystyrene capacitor to collect the charge, a potentiometer to supply and measure the bucking voltage, and a vibrating reed electrometer as a null detector. The measurements were all corrected to an air temperature of $20^{\circ} \mathrm{C}$ and a pressure of 760 $\mathrm{mm}$ of mercury. The small effect of humidity changes was eliminated by using a drier in each chamber.

The calorimeter measurements at different peak photon energies, $k_{\max }$, are listed in table 1 in units of joules absorbed by the calorimeter per monitor-volt. The rms deviations of these measurements vary from \pm 3.6 percent at an intensity of $2 \mu \mathrm{w} / \mathrm{cm}^{2}$, which required $90 \mathrm{~min}$ exposures with maximum amplifier gain, to \pm 0.7 percent at an intensity of $250 \mu \mathrm{w} /$ $\mathrm{cm}^{2}$, which required 5 min exposures with the gain reduced by a factor of 7 .

\section{Calorimeter Corrections}

The calibrations of monitors $\mathrm{A}$ and $\mathrm{B}$ were determined in joules/monitor-volt by correcting the numbers in table 1 for the fraction of incident energy which did not contribute to the calorimeter cylinder temperature rise. Part of this energy is removed from the incident beam by the calorimeter entrance foils, part is lost in nuclear transformations in the cylinder, and part escapes from the cylinder in the form of transmitted and scattered

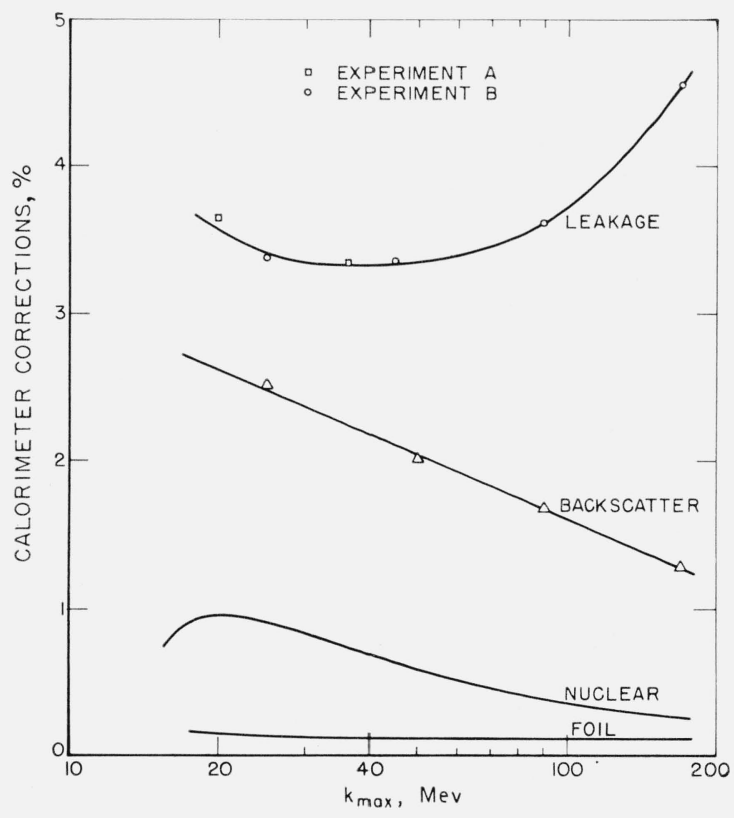

Figure 6. Corrections for energy which does not appear as heat in the calorimeter. 
TABLE 1. Calorimeter measurements of joules absorbed/monitor volt, at $20{ }^{\circ} \mathrm{C}$ and $\gamma 60 \mathrm{~mm}$ of mercury

\begin{tabular}{|c|c|c|c|c|c|c|c|c|c|c|c|c|c|c|c|c|c|c|c|c|c|}
\hline \multirow{2}{*}{$k_{\max }(\mathrm{Mev})$} & \multicolumn{7}{|c|}{ Experiment A } & \multicolumn{14}{|c|}{ Experiment $\mathrm{B}$} \\
\hline & 18.2 & 19.8 & 21.7 & 25.9 & 31.3 & 36.7 & 42.1 & 20 & 25 & 30 & 35 & 40 & 45 & 50 & 60 & 70 & 90 & 110 & 130 & 150 & 170 \\
\hline $\begin{array}{c}\text { Individual measure- } \\
\text { ments_._. }\end{array}$ & $\begin{array}{r}0.1360 \\
.1399 \\
.1381 \\
.1341 \\
.1417 \\
.1327 \\
.1326 \\
.1365 \\
.1397\end{array}$ & $\begin{array}{r}0.1463 \\
.1489 \\
.1513 \\
.1417 \\
.1527 \\
.1485 \\
.1426 \\
.1388 \\
.1500\end{array}$ & $\begin{array}{r}0.1552 \\
.1534 \\
.1620 \\
.1604 \\
.1577 \\
.1560 \\
.1526 \\
.1548 \\
.1559\end{array}$ & $\begin{array}{r}0.1671 \\
.1710 \\
.1725 \\
.1726 \\
.1733 \\
.1725 \\
.1734 \\
.1771\end{array}$ & $\begin{array}{r}0.1943 \\
.1945 \\
.1976 \\
.1993 \\
.1951 \\
.1924 \\
.1967 \\
.1957 \\
.1984\end{array}$ & $\begin{array}{l}0.2169 \\
.2161 \\
.2140 \\
.2181 \\
.2189 \\
.2165 \\
.2178 \\
.2118 \\
.2161 \\
.2138\end{array}$ & $\begin{array}{l}0.2350 \\
.2368 \\
.2431 \\
.2374 \\
.2363 \\
.2390 \\
.2387 \\
.2382 \\
.2363\end{array}$ & $\begin{array}{r}0.467 \\
.500 \\
.490 \\
.455 \\
.498 \\
.464 \\
.491 \\
.490 \\
.449 \\
.483 \\
.455 \\
.468 \\
.480\end{array}$ & $\begin{array}{r}0.590 \\
.572 \\
.591 \\
.550 \\
.611 \\
.605 \\
.555 \\
.564 \\
.557 \\
.569 \\
.602 \\
.584 \\
.600 \\
.592 \\
.579\end{array}$ & $\begin{array}{r}0.698 \\
.703 \\
.688 \\
.700 \\
.710 \\
.719 \\
.683 \\
.664 \\
.704 \\
.700 \\
.705 \\
.695 \\
.687 \\
.692\end{array}$ & $\begin{array}{r}0.799 \\
.820 \\
.790 \\
.809 \\
.788 \\
.792 \\
.800 \\
.797 \\
.801 \\
.799 \\
.782 \\
.810 \\
.804\end{array}$ & $\begin{array}{r}0.892 \\
.906 \\
.861 \\
.901 \\
.884 \\
.887 \\
.883 \\
.855 \\
.900 \\
.901 \\
.894 \\
.889 \\
.877\end{array}$ & $\begin{array}{r}0.961 \\
.957 \\
.984 \\
.976 \\
.976 \\
.966 \\
.980 \\
.981 \\
.968 \\
.967 \\
.966 \\
.975\end{array}$ & $\begin{array}{l}1.052 \\
1.087 \\
1.050 \\
1.051 \\
1.070 \\
1.065 \\
1.061 \\
1.068 \\
1.058 \\
1.061 \\
1.048 \\
1.051\end{array}$ & $\begin{array}{l}1.200 \\
1.198 \\
1.221 \\
1.221 \\
1.206 \\
1.232 \\
1.233 \\
1.214 \\
1.225 \\
1.223\end{array}$ & $\begin{array}{l}1.387 \\
1.361 \\
1.371 \\
1.358 \\
1.354 \\
1.288 \\
1.305 \\
1.281 \\
1.283 \\
1.376 \\
1.363 \\
1.358 \\
1.353 \\
1.362 \\
1.354 \\
1.365 \\
1.377 \\
1.364\end{array}$ & $\begin{array}{l}1.632 \\
1.677 \\
1.588 \\
1.587 \\
1.622 \\
1.579 \\
1.584 \\
1.596 \\
1.583 \\
1.580 \\
1.530 \\
1.616 \\
1.612 \\
1.626 \\
\end{array}$ & $\begin{array}{l}1.825 \\
1.821 \\
1.835 \\
1.899 \\
1.893 \\
1.820 \\
1.844 \\
1.831\end{array}$ & $\begin{array}{l}2.047 \\
2.044 \\
2.038 \\
2.028 \\
2.036 \\
2.026 \\
2.018 \\
2.010 \\
2.014 \\
2.003\end{array}$ & $\begin{array}{l}2.219 \\
2.214 \\
2.193 \\
2.217 \\
2.207 \\
2.202 \\
2.187 \\
2.180 \\
2.167 \\
2.197\end{array}$ & $\begin{array}{l}2.383 \\
2.374 \\
2.366 \\
2.373 \\
2.380 \\
2.364 \\
2.324 \\
2.342 \\
2.348\end{array}$ \\
\hline Averages _........... & .1366 & .1467 & .1564 & .1724 & .1960 & .2160 & .2379 & .476 & .581 & .696 & .799 & .887 & .971 & 1. 060 & 1. 217 & 1.348 & 1. 601 & 1. 846 & 2.026 & 2. 198 & 2. 362 \\
\hline Deviations, Percent rms. & 2.3 & 3.2 & 2.0 & 1. 6 & 1.1 & 1.0 & 1.0 & 3.6 & 3.3 & 1.9 & 1.3 & 1. 7 & 0.9 & 1. 0 & 1.0 & 2.5 & 2.1 & 1. 7 & 0.7 & 0.8 & 0.8 \\
\hline
\end{tabular}

TABLE 2. Absolute calorimetric calibration of $P 2-4$ at $20{ }^{\circ} \mathrm{C}$ and $760 \mathrm{~mm}$ of mercury in a $4.2 \mathrm{~cm}$ diam beam filtered by $4.5 \mathrm{~g} / \mathrm{cm}^{2}$ of aluminum

\begin{tabular}{|c|c|c|c|c|}
\hline \multicolumn{5}{|c|}{ Experiment A } \\
\hline$k_{\max }$ & $\frac{\text { Joules }}{\text { mon. volt }}$ & $\frac{P^{2}-4 \text { volts }}{\text { mon. volt }}$ & $F_{A}$ & $\mathrm{Cal}(\mathrm{P} 2-4)$ \\
\hline $\begin{array}{l}\text { Mev } \\
18.2 \\
19.8 \\
21.7 \\
25.9 \\
31.3 \\
36.7 \\
42.1\end{array}$ & $\begin{array}{r}0.1472 \\
.1579 \\
.1680 \\
.1848 \\
.2094 \\
.2305 \\
.2536\end{array}$ & $\begin{array}{l}3.442 \\
3.693 \\
3.941 \\
4.361 \\
4.962 \\
5.495 \\
6.002\end{array}$ & $\begin{array}{r}0.998 \\
.998 \\
.998 \\
.998 \\
.999 \\
.999 \\
.999\end{array}$ & 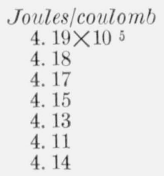 \\
\hline
\end{tabular}

Experiment B

\begin{tabular}{r|l|l|l|l}
\hline 20 & 0.512 & 12.20 & 1 & $4.12 \times 10^{5}$ \\
25 & .623 & 14.97 & 1 & 4.08 \\
30 & .744 & 17.74 & 1 & 4.11 \\
35 & .853 & 20.38 & 1 & 4.10 \\
40 & .946 & 22.88 & 1 & 4.05 \\
45 & 1.034 & 25.25 & 1 & 4.02 \\
50 & 1.128 & 27.70 & 1 & 3.99 \\
60 & 1.293 & 32.18 & 1 & 3.94 \\
70 & 1.431 & 36.31 & 1 & 3.86 \\
90 & 1.696 & 43.80 & 1 & 3.80 \\
110 & 1.956 & 50.00 & 1 & 3.84 \\
130 & 2.151 & 55.39 & 1 & 3.81 \\
150 & 2.336 & 59.90 & 1 & 3.82 \\
170 & 2.513 & 63.73 & 1 & 3.87 \\
\hline
\end{tabular}

Average

\begin{tabular}{|c|c|c|c|}
\hline \multirow{2}{*}{$k_{\max }$} & \multirow{2}{*}{$\mathrm{Cal}(\mathrm{P} 2-4)$} & \multicolumn{2}{|c|}{ Percent error } \\
\hline & & $\mathrm{sdm}$ & Total \\
\hline $\begin{array}{r}M e v \\
20 \\
26 \\
31 \\
36 \\
41 \\
45 \\
50 \\
60 \\
70 \\
90 \\
110 \\
130 \\
150 \\
170\end{array}$ & $\begin{array}{l}\text { Joules/ } \\
\text { coulomb } \\
\text { 4. } 16 \times 10^{5} \\
4.11 \\
4.12 \\
4.11 \\
4.09 \\
4.02 \\
3.99 \\
3.94 \\
3.86 \\
3.80 \\
3.84 \\
3.81 \\
3.82 \\
3.87\end{array}$ & $\begin{array}{r}0.5 \\
.6 \\
.3 \\
.2 \\
.4 \\
.3 \\
.3 \\
.3 \\
.6 \\
.6 \\
.6 \\
.2 \\
.3 \\
.3\end{array}$ & $\begin{array}{l}2.1 \\
2.4 \\
1.5 \\
1.2 \\
1.8 \\
1.5 \\
1.5 \\
1.5 \\
2.4 \\
2.4 \\
2.4 \\
1.2 \\
1.5 \\
1.5\end{array}$ \\
\hline
\end{tabular}

photons and secondary electrons. ${ }^{2}$ The determination of these corrections is described below, and the corrections are plotted in figure 6 as a function of bremsstrahlung peak photon energy. The corrected monitor calibrations are listed in table 2 for both experiments.

\subsection{Calculated Corrections}

The energy removed by the calorimeter entrance foils and by nuclear transformations was calculated as a function of photon energy and averaged over the bremsstrahlung spectra obtained from the tabulations of reference [8], corrected for filtration with the total attenuation coefficients of reference [9].

The probability of an interaction in the foils which removed a photon of energy $k$ from the beam incident on the calorimeter cylinder was taken to be $1 / 2 \alpha(k) t$, where $t$ is the total foil thickness $\left(0.1 \mathrm{~g} / \mathrm{cm}^{2}\right.$ of aluminum), and $\alpha(k)$ is the total attenuation coefficient of aluminum [9]. The factor $1 / 2$ represents an estimate of the probability that the interaction products will not strike the cylinder. The foil correction is so small that large errors in this estimate are unimportant.

Only $(\gamma, n)$ reactions were considered in calculating the nuclear correction since $(\gamma, p)$ reactions are much less probable. The probability for a $(\gamma, n)$ reaction was taken as the triple product of the atomic density of lead $\left(2.91 \times 10^{21}\right.$ atoms $\left./ \mathrm{g}\right)$, the reaction cross section $\left(\mathrm{cm}^{2}\right)$ and the effective length of the cylinder $\left(\mathrm{g} / \mathrm{cm}^{2}\right)$. The cross section was taken from reference [10] below $18 \mathrm{Mev}$ and from reference [11] at higher

2 The amount of energy stored by the lead crystal when lead atoms are displaced to interstitial sites by X-rays and secondary electrons in the cylinder can be shown to be negligible: The cross section for atomic displacement in lead is of the order of $3 \times 10^{-24} \mathrm{~cm}^{2}$ for X-rays and $10^{-22} \mathrm{~cm}^{2}$ for electrons, and each displaced order of $3 \times 10^{-24} \mathrm{~cm}^{2}$ for $X$-rays and $10^{-22} \mathrm{~cm}^{2}$ for electrons, and each displaced
atom will in turn displace no more than five additional atoms [7]. Each disatom will in turn displace no more than five additional atoms [7]. Each displacement results in the storage of less than $25 \mathrm{ev}$. In traveling one mean-free
path, an X-ray of any energy loses no more than 0.002 percent of its energy to the path, an X-ray of any energy loses no more than 0.002 percent of its energy to the
crystal. The energy lost to the crystal by an electron, in traveling $1 \mathrm{~cm}$, is less than 0.005 percent of the normal energy loss rate at all energies. 
energies. The effective length of the cylinder is $\left(1-e^{-\beta L}\right) / \beta$, where $L$ is the true length and $\beta$ is an attenuation coefficient describing the decrease of beam intensity with increasing depth in the cylinder. In the initial calculations, $\beta$ was taken from tabulations of total attenuation coefficients [9], which underestimate the effective length because their use assumes that all interaction products are removed from the beam. In a second set of calculations, an average $\beta$ was taken from the slope of experimental transition curves discussed in the following section (fig. 9). These $\beta$ overestimate the effective length because they are close to the attenuation coefficient for minimum absorption, which occurs at about $4 \mathrm{Mev}$ in lead. The average photon energy for $(\gamma, n)$ reactions is larger than this and the average $\beta$ should also be larger. However, at each energy, the fraction of energy lost to $(\gamma, n)$ reactions predicted by these two sets of $\beta$ 's differed by less than $1 / 4$ percent and the average effective length was used in the final calculations.

The amount of energy removed from the incident beam by a $(\gamma, n)$ reaction was taken to be the neutron binding energy in lead, $7.9 \mathrm{Mev}$ [10], plus an average neutron kinetic energy of $2.6 \mathrm{Mev}$ [12].

\subsection{Measured Corrections}

The leakage of photons and electrons from the surfaces of the calorimeter cylinder was investigated in two subsidiary experiments. The first was performed by replacing the calorimeter cylinder by a large lead medium and determining the relative amount of energy, $e(r, x)$, absorbed at different

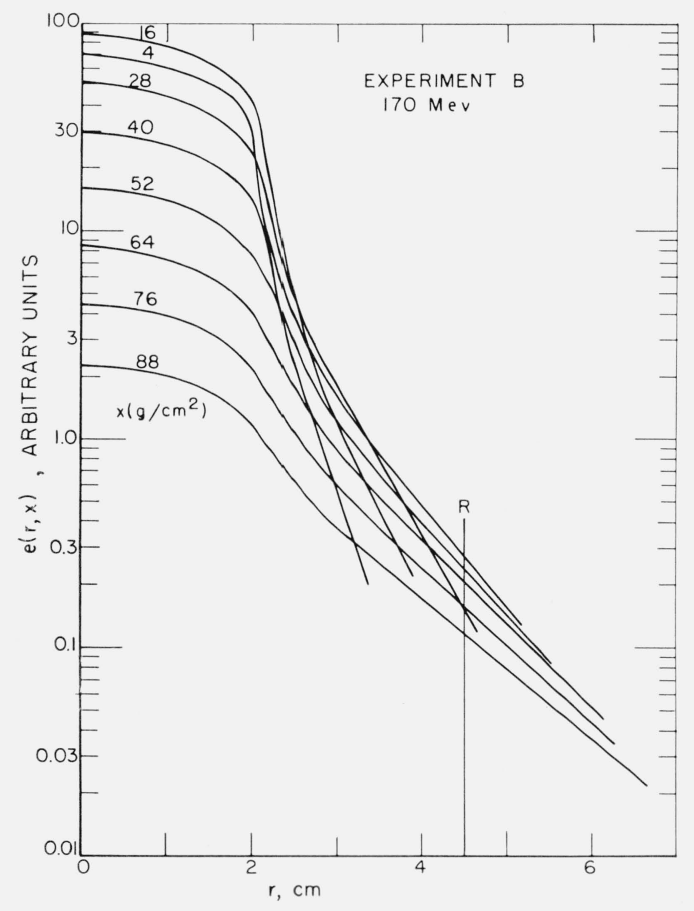

Figure 7. Typical radial distributions of absorbed energy at several depths in a lead medium.

$R$ is the radius of the calorimeter cylinder. points, where $r$ is radius, measured from the beam axis, and $x$ is depth in the medium. The medium was composed of $24 \mathrm{~cm}$ diam lead disks mounted perpendicular to the beam axis, each $12 \mathrm{~g} / \mathrm{cm}^{2}$ thick. $e(r, x)$ was obtained from densitometer measurements of X-ray films which had been sandwiched between these disks and exposed to X-rays. The analysis assumed that density was proportional to absorbed energy, an assumption which was justified in the tests described at the end of this section. Figure 7 shows the densitometer traces obtained at $170 \mathrm{Mev}$ in experiment B, normalized to the same energy incident on the medium.

The fraction of energy absorbed in the medium outside the cylinder volume is:

$$
\text { leakage fraction }=\frac{\int_{0}^{L} I_{1}(x) d x+\int_{L}^{\infty} I_{2}(x) d x}{\int_{0}^{\infty} I_{2}(x) d x}
$$

where:

$$
\begin{aligned}
& I_{1}(x)=\int_{R}^{\infty} e(r, x) r d r \\
& I_{2}(x)=\int_{0}^{\infty} e(r, x) r d r
\end{aligned}
$$

and $R$ and $L$ are the radius and length of the cylinder, respectively. The integrals $I_{1}(x)$ and $I_{2}(x)$ are plotted in figures 8 and 9 for the data obtained in experiment B. The curves of figure 9 are broadbeam transition curves, showing the relative amount of energy which would be absorbed at different depths in a lead medium bombarded by a uniform bremsstrahlung beam of infinite diameter.

The leakage fraction predicted by eq (1) is smaller than the fraction of energy leaking from the sides and back of the calorimeter cylinder in vacuo because of the enhanced backscatter of the lead medium at the cylinder boundaries. However, it was shown that the difference is negligible by exposing films at depth $L$ with different thicknesses of backing. As the backing thickness was increased from zero, the absorbed energy rose to a saturation value at a thickness of only $1 \mathrm{~mm}$.

The second subsidiary experiment was a determination of the fraction of energy backscattered from the calorimeter cylinder itself. This experiment was performed with a scintillation spectrometer, using a cylindrical sodium iodide crystal with an axial hole, mounted in front of the calorimeter cylinder as shown in figure 10. The crystal was viewed by four photomultipliers, which generated an electronic pulse each time a backscattered photon or secondary electron interacted with the crystal. A 256 channel pulse height analyzer was used to sort and display the pulses as a function of pulse height. Figure 11 shows the pulse height distributions obtained at $25,50,90$, and $170 \mathrm{Mev}$, corrected for background. In these distributions, the large peaks at $511 \mathrm{kev}$ were produced by photons resulting from positron annihilation in the cylinder, 


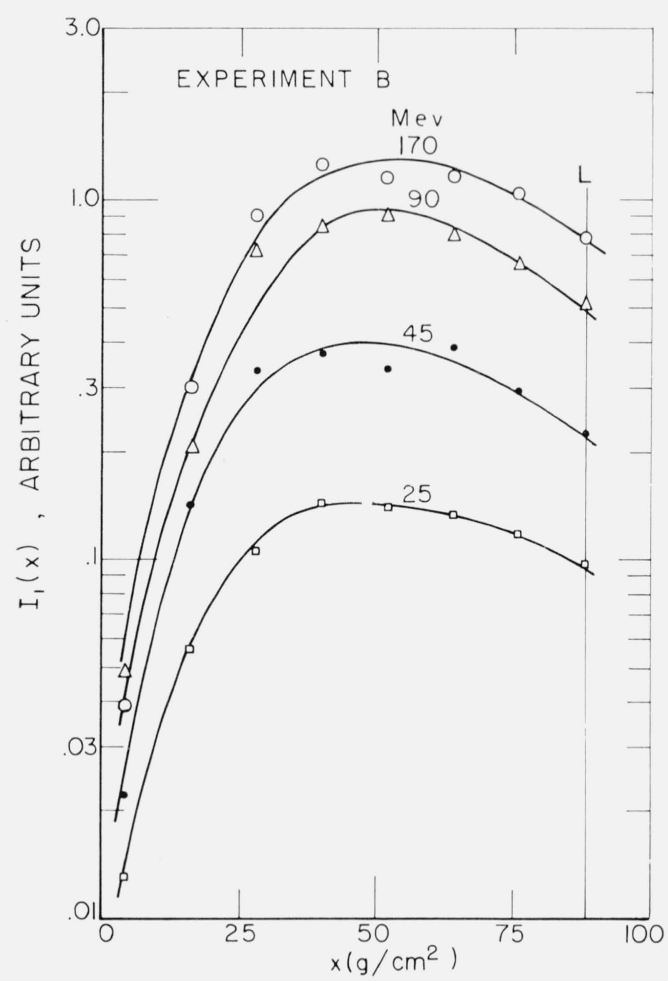

Figure 8. Sidescattered energy. $I_{1}(x)=\int_{R}^{\infty} e(r, x) r d r$ from the film measurements of experiment B. $L$ is the length of the calorimeter cylinder.

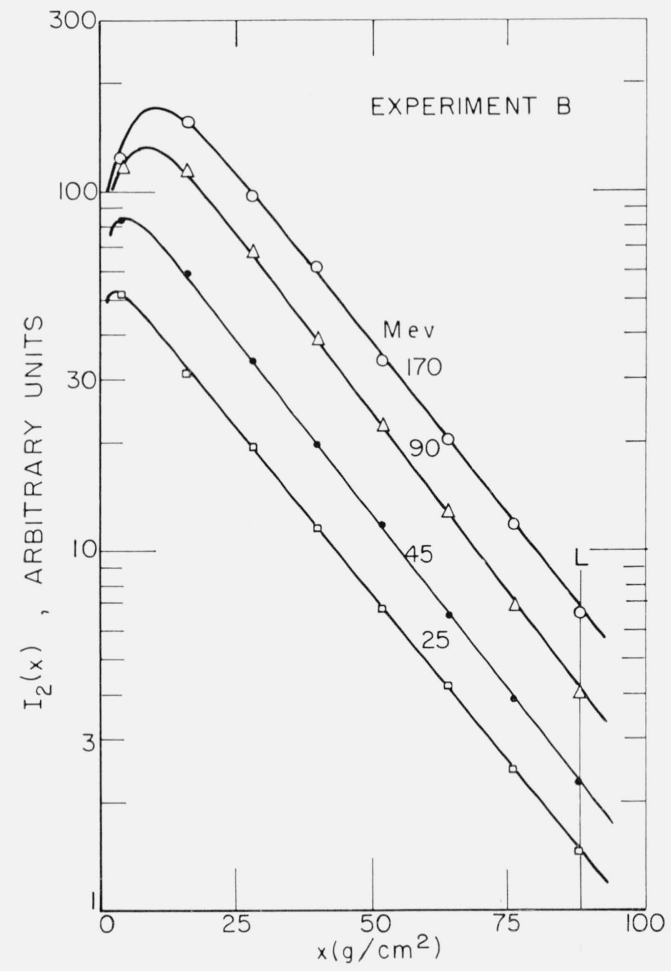

Figure 9. Transition curves.

$I_{2}(x)=\int_{0}^{\infty} e(r, x) r d r$ from the film measurements of experiment B. $L$ is the length of the calorimeter cylinder.

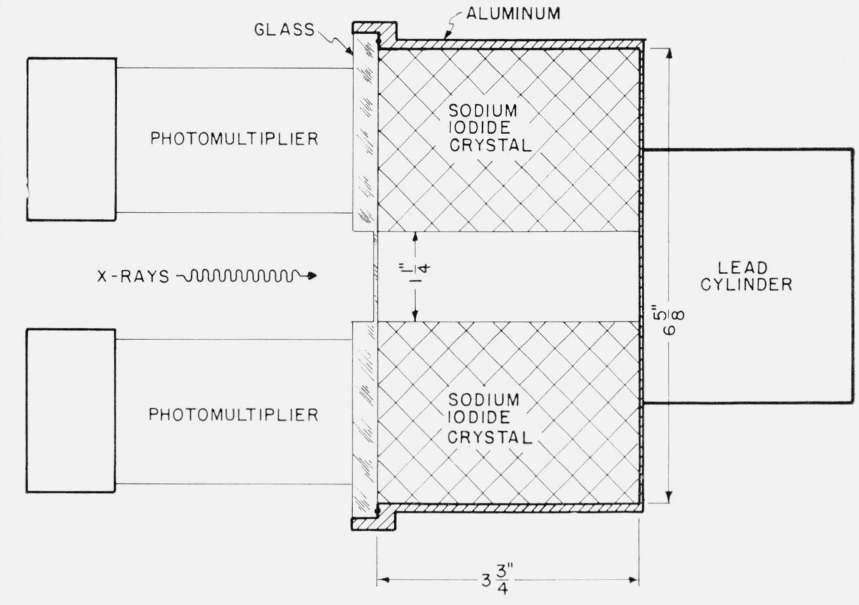

FiguRE 10.-Arrangement of scantillation spectrometer to determine fraction of energy backscattered from calorimeter cylinder.

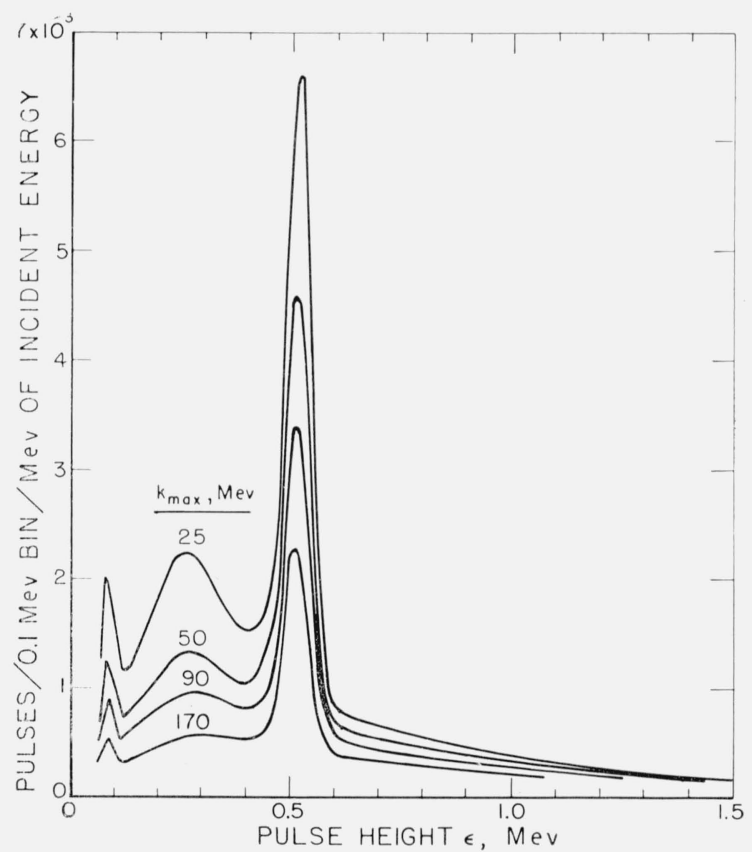

Figure 11. Pulse height spectra produced by radiation backscattered from calorimeter cylinder.

the broad peaks around $250 \mathrm{kev}$ were produced by singly-scattered photons, and the peaks at $80 \mathrm{kev}$ by lead $K$ X-rays. The low level background, which decreases monotonically with increasing pulse height, $\epsilon$, was presumably caused by electrons, multiply-scattered photons, and bremsstrahlung.

Each pulse height distribution, $P(\epsilon)$, was transformed into a particle spectrum, $N(k)$, by solving the integral equation:

$$
P(\epsilon)=\int_{0}^{k_{\max }} N(k) S(k) K(k, \epsilon) d k
$$

where $S(k)$ is the probability that a photon or electron of energy $k$ will interact with the crystal, and 


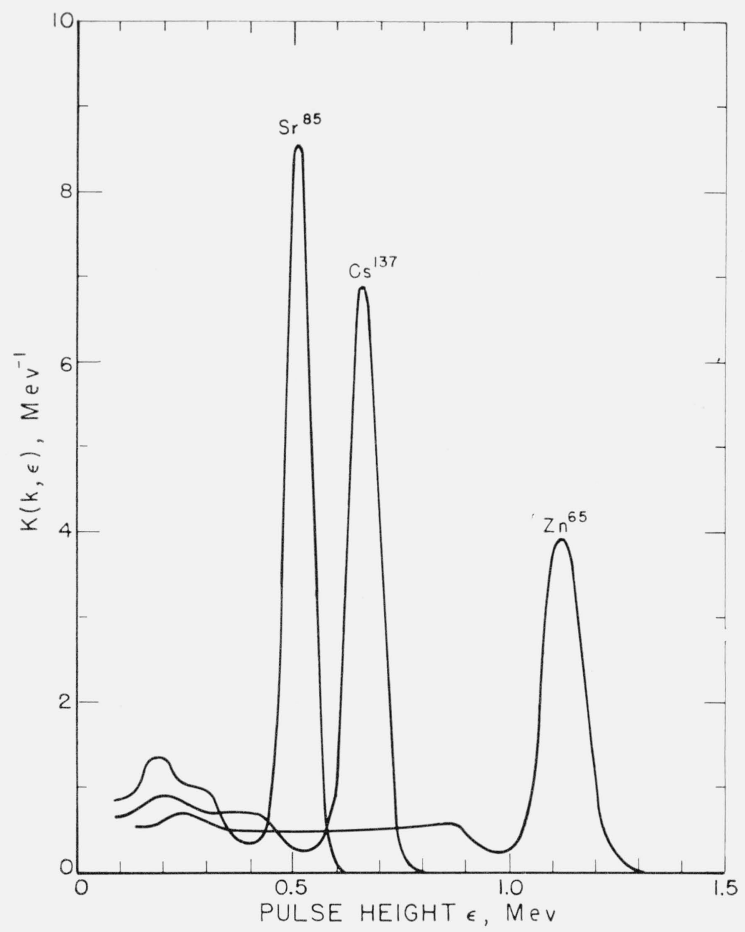

Figure 12. Pulse height spectra produced in the backscatter crystal by monoenergetic photons from radioactive sources, normalized to unit enclosed area.

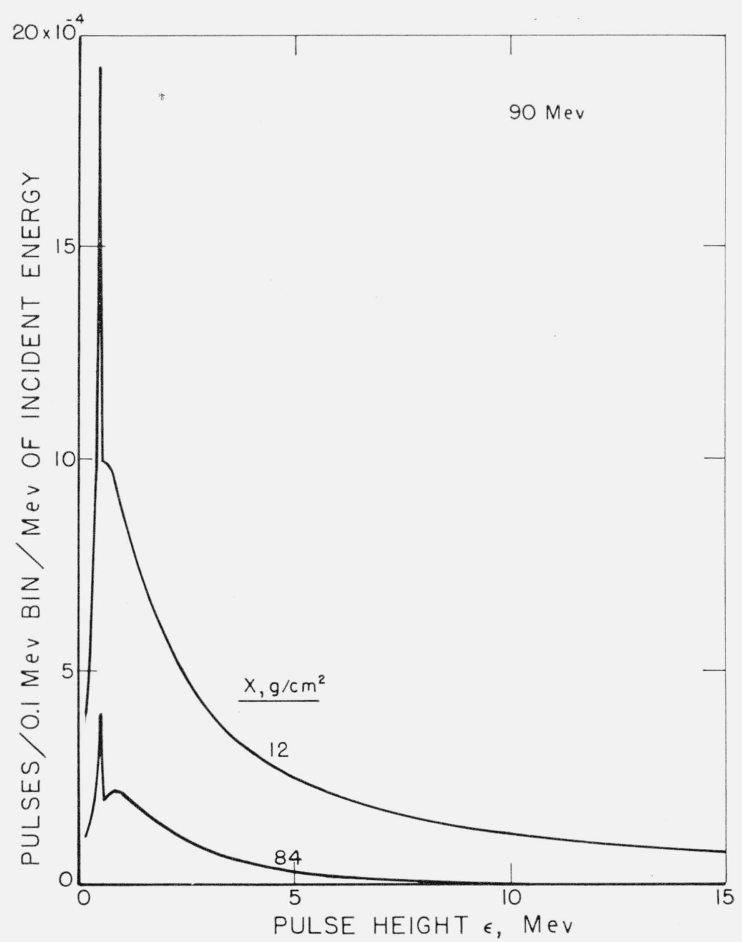

Figure 13. Pulse height spectra produced by the 90 Mev bremsstrahlung transmitted by a lead medium of thickness $X$.
$K(k, \epsilon)$ is the probability that it will generate a pulse of height $\epsilon$ after the initial interaction. For given $k, K(k, \epsilon)$ is the pulse height distribution produced by photons or electrons of that energy, normalized to unit enclosed area.

The scintillation spectrometer cannot distinguish between incident photons and electrons, yet the transformation from $P(\epsilon)$ to $N(k)$ depends upon the nature of the incident particles because both the sensitivity, $S(k)$, and the response function, $K(k, \epsilon)$, differ for photons and electrons. The transformation was first made by assuming that only photons were incident on the crystal and solving eq (2) by standard matrix methods [13]. $K(k, \epsilon)$ was measured for $k=0.51,0.66$, and $1.12 \mathrm{Mev}$ by placing $\mathrm{Sr}$, Cs, and $\mathrm{Zn}$ radioactive sources on the front surface of the cylinder, producing the pulse height distributions shown in figure 12. Each of these distributions was approximated by a one parameter function, a delta function at $\epsilon=\dot{k}$ and a flat tail for $\epsilon<k$. The one parameter, the relative area under the delta function, was measured from the distributions of figure 12 at low energies and was extrapolated to higher energies with the help of the theoretical predictions of Berger and Doggett [14]. This relative area, or "photofraction," varied from 0.96 at $0.3 \mathrm{Mev}$ to 0.08 at 170 Mev. $S(k)$ was calculated from the crystal geometry, assuming isotropic backscattering, and using published total attenuation coefficients for sodium iodide [9]. It varied from 0.985 below $0.1 \mathrm{Mev}$ to a minimum of 0.580 at $5 \mathrm{Mev}$. The fraction of energy backscattered was calculated from:

$$
\text { fraction backscattered }=\int_{0}^{k_{\max }} k N(k) d k .
$$

The fraction backscattered was recalculated assuming that only electrons were incident on the crystal, using $K(k, \epsilon)=\delta(k-\epsilon)$ and $S(k)=0.985$ for all $k$. These two determinations of the fraction backscattered were weighted and averaged, assuming that $13 \frac{1}{2}$ percent of the backscattered energy was carried by electrons. This estimate is one-half of the fraction of the energy emerging from the back of the cylinder which is carried by electrons, as revealed by the shape of the transition curves of figure 9 with the help of a simple theory of energy absorption [15]. This method of estimating the electron contribution is only approximate, but since it reduced the fraction calculated for photons by only about 0.2 percent at all energies, the uncertainties are relatively unimportant.

The scintillation spectrometer was also used to check the film measurements of the variation of absorbed energy with position in a lead medium. This was done with a $9 \mathrm{in}$. diam, $6 \frac{1}{4} \mathrm{in}$. long sodium iodide crystal placed immediately behind the medium to look at the spectrum of transmitted X-ravs and electrons. Figure 13 shows the pulse height distributions obtained with 90 Mev bremsstrahlung, using two different medium thicknesses. These distributions, plus one at $25 \mathrm{Mev}$, were analyzed to find the fraction of energy transmitted by the medium, 
using the analytical technique outlined above. The results are listed in table 3 along with the predictions of the film measurements, which come from the formula:

$$
\text { fraction of energy transmitted }=\frac{\int_{X}^{\infty} I_{2}(x) d x}{\int_{0}^{\infty} I_{2}(x) d x}
$$

where $X$ is the medium thickness. The good agreement between these two determinations of the transmitted energy was interpreted as a verification of the film analysis procedure.

TABLE 3. Comparison of film and spectrometer determinations of energy transmitted by lead medium of thickness $X$

\begin{tabular}{c|c|c|c}
\hline \hline & & \multicolumn{2}{|c}{ Percent transmitted } \\
\cline { 3 - 3 } & & $X$ & \multicolumn{2}{|c}{ Film } \\
\cline { 3 - 4 } & & Spectrometer & Film \\
\hline $90 v$ & $g / \mathrm{cm}^{2}$ & & \\
\hline 90 & 12 & 64.1 & 59.3 \\
$25 \ldots$ & 84 & 2.85 & 2.64 \\
\hline
\end{tabular}

\section{Ionization Chamber Calibrations}

The monitor calibrations of table 2 have only a transitory value, and were transferred by direct comparison to the more permanent aluminum ionization chamber shown in figure 14. This large chamber is labeled P2-4 and has been described in detail elsewhere [16]. Its calibration in joules/ coulomb was obtained from the formula:

$$
\text { Cal }(\mathrm{P} 2-4)=\frac{(\text { Joules } / \text { monitor volt }) \times F_{A}}{C \times(\mathrm{P} 2-4 \text { volts } / \text { monitor volt })}
$$

where $C$ is the capacitance of the capacitors charged by $\mathrm{P} 2-4 \quad\left(0.10197 \times 10^{-6} \pm 0.05 \%\right.$ farads $)$ and $F_{A}$ is a small correction [16] used with the data of experiment $A$ to change it to refer to the filtration of experiment B. ${ }^{3}$

The calibrations of $\mathrm{P} 2-4$ are listed in table $2,{ }^{4}$ along with $F_{A}$ and the chamber comparison data.

The P2-4 calibrations from experiments $\mathrm{A}$ and B agree to within 2 percent except for the $42.1 \mathrm{Mev}$ measurement, which is a trifle high. The data from the two experiments were combined, to yield better statistics, and the average calibrations are also listed in table 2. Data from the three lowest energies in experiment A were averaged with the 20 Mev data in experiment $B$, but otherwise the energies were paired, below $45 \mathrm{Mev}$. This averaging introduces some uncertainty in the energy assignments,

\footnotetext{
3 The filtration of experiment B is listed as entirely of aluminum, since the calibrations are insensitive to the difference between Pyrex and aluminum filters [16].

4 The earlier versions of these calibrations which appeared in references [5] and [18] differ because they were printed before the backscatter correction had been determined.
}

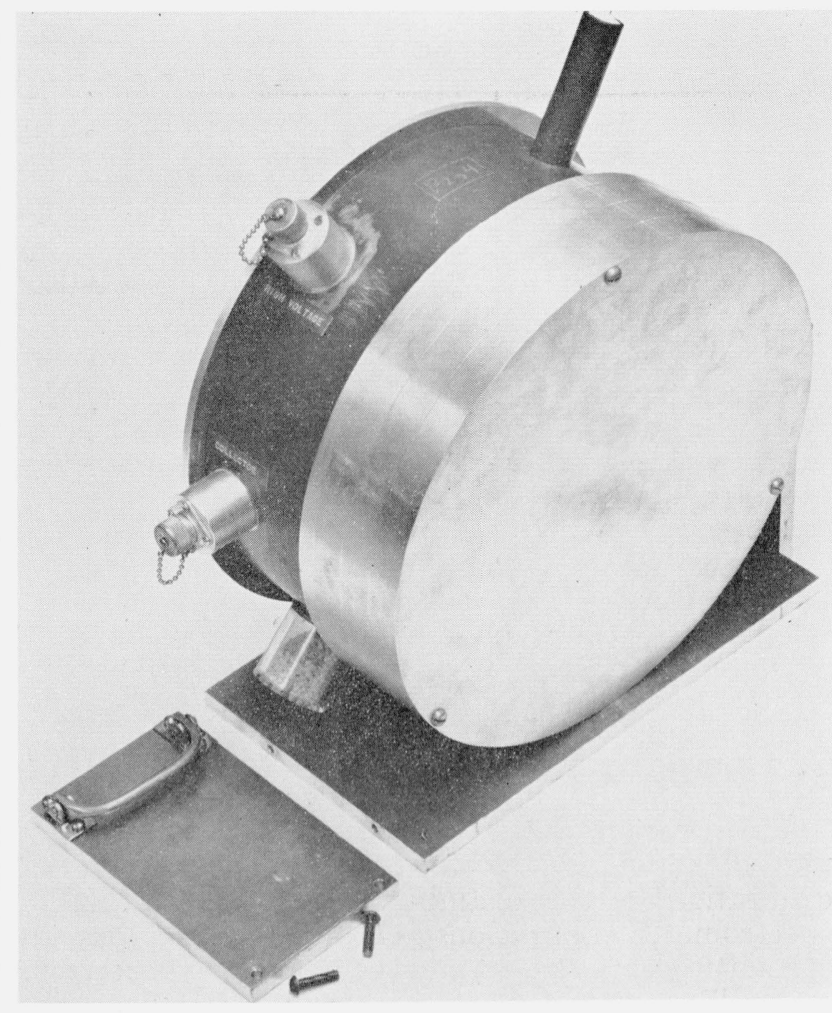

Figure 14.-Aluminum ionization chamber P2-4.

but this is inconsequential because the calibration varies slowly with $k_{\max }$ below $45 \mathrm{Mev}$. The numbers listed as sdm in table 2 are the statistical standard deviations of the mean of each average:

$$
\operatorname{sdm}=\sqrt{\frac{\sum_{i} \delta_{i}^{2}}{n(n-1)}}
$$

where $\delta_{i}$ is a deviation from the mean and $n$ is the number of cases. The net systematic error was taken to be the square root of the sum of the squares of the estimated maximum systematic errors listed in table 4 . The statistical and systematic errors were combined with the arbitrary formula:

Total error $=3 \times$ sdm + net systematic error.

The statistical part of the total error of each point is consequently considerably more pessimistic than a probable error.

\begin{tabular}{|c|c|}
\hline $\begin{array}{l}\text { Electrical energy measurements } \\
\text { Monitor stability } \\
\text { Foil correction } \\
\text { Nuclear correction } \\
\text { Leakage correction } \\
\text { Backscatter correction }\end{array}$ & $\begin{array}{r}\% \\
0.2 \\
.2 \\
.1 \\
.2 \\
.3 \\
.3\end{array}$ \\
\hline Net systematic error & .6 \\
\hline
\end{tabular}

TABLE 4. Estimated maximum systematic errors in $\mathrm{P}_{2}-4$ calibration 


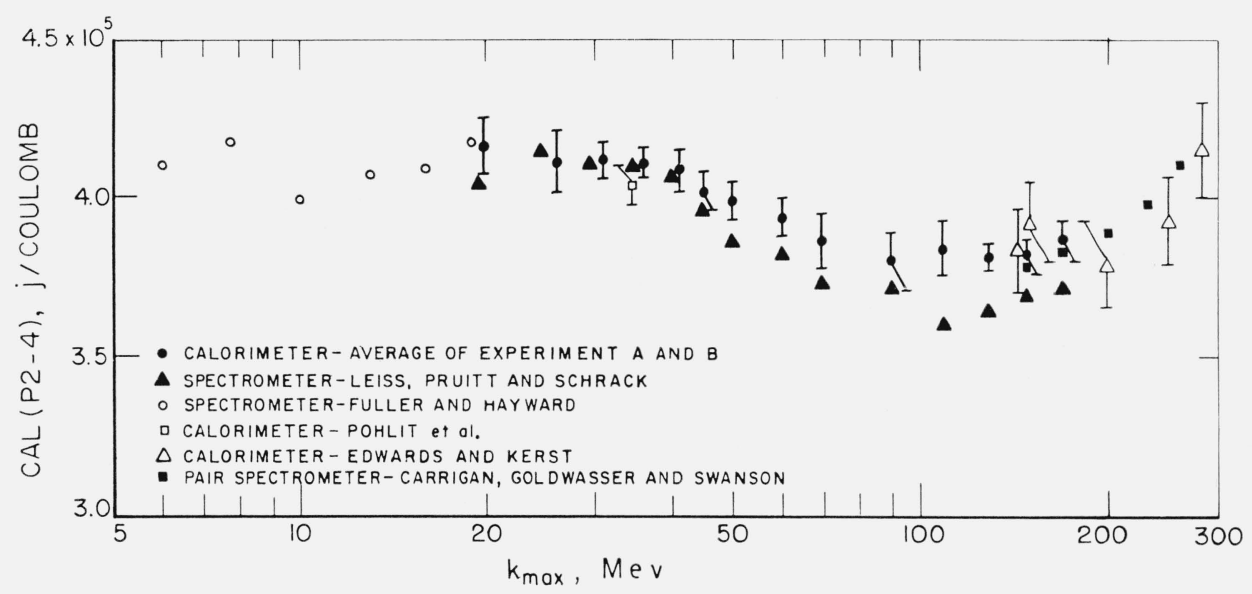

Figure 15. Absolute calibrations of ionization chamber P2-4 in a $4.2 \mathrm{~cm}$ diam bremsstrahlung beam filtered by $4.5 \mathrm{~g} / \mathrm{cm}^{2}$ of aluminum, at $20^{\circ} \mathrm{C}$ and $760 \mathrm{~mm}$ of mercury.

\section{Comparison With Other Measurements}

The average P2-4 calibrations of table 2 are plotted in figure 15 for comparison with other calibrations of this chamber. The first scintillation spectrometer calibrations [17] were assigned an uncertainty of \pm 3 percent and the more recent spectrometer calibrations [18] an uncertainty of \pm 2 percent. The $34.5 \mathrm{Mev}$ calorimetric calibration was made with chamber P2-6 in Frankfurt and was transferred to $\mathrm{P} 2-4$ by direct comparison [5]. The calorimetric calibrations based on the work of P. D. Edwards and D. W. Kerst [3] were transferred to P2-4 from replicas of their copper ionization chamber at the NBS and the University of Illinois. The pair spectrometer measurements [19] are not absolute, and were normalized to a weighted average of the NBS calorimeter and spectrometer calibrations at 170 Mev. The NBS spectrometer calibrations and the Frankfurt calibration have been corrected for differences in beam diameter and filtration [16]. No such corrections have been applied to the other points, but they are probably quite small compared to the uncertainties in these points.

The P2-4 calibrations shown in figure 15 are all in good agreement except for the spectrometer measurements above $45 \mathrm{Mev}$, which differ from the calorimeter measurements by as much as 5 percent. The source of this discrepancy is thought to be a time dependent systematic error in the spectrometer measurements, which were made in two sets. The measurements above $45 \mathrm{Mev}$ predated the lower energy measurements, except at $90 \mathrm{Mev}$, which contains data from both sets. This is the high energy spectrometer point in closest agreement with the calorimeter calibrations.

The authors express their thanks to all members of the NBS High Energy Radiation Section for their help with these experiments, and to J.
McElhinney and B. Zendle for their invaluable pioneering work.

\section{References}

[1] NBS Handbook 55, appendix B (1954); H. W. Koch, J. E. Leiss, and J. S. Pruitt, Bull. APS II 1, 199 (1956) A; R. R. Wilson, Nuclear Instr. 1, 101 (1957).

[2] J. S. Laughlin and J. W. Beattie, Rev. Sci. Instr. 22, 575 (1951); Laughlin, Beattie, Henderson, and Harvey, Am. J. Roentgenol. \%o, 294 (1953); Skarsgard, Bernier, Cormack, and Johns, Rad. Research $\boldsymbol{7}, 217$ (1957); G. D. Adams, Radiology 69, 867 (1957).

[3] P. D. Edwards and D. W. Kerst, Rev. Sci. Instr. 24, 490 (1953).

[4] S. P. Kruglov, Zhur. Tekh. Fiz. 28, 2310 (1958) (Soviet Phys.-Tech. Phys. 3, 2120 (1958)); S. P. Kruglov and I. V. Lopatin, Zhur. Tekh. Fiz. 30, 424 (1960).

[5] J. S. Pruitt and W. Pohlit, Z. Naturforsch. 15b, 617 (1960)

[6] J. McElhinney, B. Zendle, and S. R. Domen, J. Research NBS 56, 9 (1956).

[7] F. Seitz and J. S. Koehler, Proc. of Int. Conf. on Peaceful Uses of Atomic Energy, United Nations (1956), Vol. 7 , p. 615 .

[8] A. S. Penfold and J. E. Leiss, Analysis of photo cross sections, (Phys. Research Lab., Univ. of Illinois, Champaign, Ill., 1958).

[9] G. W. Grodstein, NBS Circular 583 (1957).

[10] R. Montalbetti, L. Katz, and J. Goldemberg, Phys. Rev. 91, 659 (1953).

[11] L. W. Jones and K. M. Terwilliger, Phys. Rev. 91, 699 (1953)

[12] M. E. Toms and W. E. Stephens, Phys. Rev. 108, 77 (1957); W. R. Dixon, Can. J. Phys. 33, 785 (1955).

[13] J. Kockum and N. Starfelt, Nuclear Instr. and Meth. 4, 171 (1959).

[14] M. J. Berger and J. Doggett, J. Research NBS 56, 355 (1956).

[15] F. Rasetti, Elements of nuclear physics, p. 96, eq. III, 34. (Prentice-Hall, New York, 1936).

[16] J. S. Pruitt and S. R. Domen, NBS Monograph 48 (1962).

[17] J. E. Leiss, J. S. Pruitt, and R. A. Schrack (unpublished data).

[18] E. G. Fuller and Evans Hayward, J. Research NBS 65A, (Phys. and Chem.) No. 5, 401 (1961).

[19] R. Carrigan, E. Goldwasser, and W. Swanson (private communication).

(Paper 66A5-172) 